\title{
Notes on bees (Hymenoptera: Apoidea: Apiformes) of central Poland
}

\author{
Józef BANASZAK* and Jan Krzysztof KowALCZYK** \\ *Institute of Biology and Environmental Protection, Kazimierz Wielki University, Chodkiewicza 30, \\ 85-064 Bydgoszcz, Poland \\ **Natural History Museum, University of Łódź, Kilińskiego 101, 90-011 Łódź, Poland
}

\begin{abstract}
A list of 233 species of bees (Apiformes) in central Poland is presented. The lowland, largely deforested part of the study area (Łódź Hills), was dominated by Heriades truncorum ( $4.3 \%$ of total catch) and Hylaeus communis (2.7\%), while the wooded Świętokrzyskie Mountains were dominated by bumblebees, particularly by Bombus lucorum (4.2\%) with its cleptoparasite Psithyrus bohemicus (6.8\%), as well as Bombus pascuorum (3.7\%), Bombus lapidarius (2.4\%) and Psithyrus campestris (2.4\%).
\end{abstract}

Key words: Hymenoptera, Apoidea, Apiformes, central Poland, occurrence of species

\section{INTRODUCTION}

This work is a contribution to the knowledge about the occurrence of bees (Apiformes) of central Poland and the Świętokrzyskie Mountains. We assumed that central Poland is the area within a 100-km radius of the Łódź city. It lies at the borders between three geographic regions: Małopolska (Little Poland), Wielkopolska (Great Poland), and Mazovia. This area is intermediate between the uplands of southern Poland and the lowlands of northern Poland. The middle part of the study area is occupied by the Lódź Hills. Until the early $20^{\text {th }}$ century, they were covered by the Łódź Forest, with a large share of beech and fir in the tree layer and of montane plant species in the herb layer (Zaręba 1981). At present anthropogenic landscapes dominate there, and the Łódź agglomeration extends at the western part of the hills. Remnants of the primeval forest are now protected within nature reserves and some urban parks, but woodlands cover only $16 \%$ of the study area. It is noteworthy that Łódź lies at the watershed between catchments of the rivers Vistula and Odra (Oder), so there are only small springs, streams and small rivers. Although the history of research on the Apoidea started nearly 200 years ago, paradoxically the central part of our country is relatively poorly studied. Among older publications on this subject, Drogoszewski's $(1932,1934)$ studies are noteworthy. He listed 178 species of Apidae from Łowicz (in the Mazovian Lowland) and 98 species from the Świętokrzyskie Mts.

Over the last 30 years, the Łódź city and the Łódź Hills were systematically studied by J. K. Kowalczyk, but bees until recently were mentioned in only several of his publications (Kowalczyk \& Watała 1987, 1990; Kowalczyk 1996; Kowalczyk et al. 1998; Szczepko et al. 2004). A list of 127 species of Apiformes from the Botanical Garden in Łódź was presented by Kowalczyk et al. (2004) and Kowalczyk \& Kurzac (2005). In the part of the Mazovian Lowland located within the borders of our study area, 54 species of Apoidea have been recorded recently in the Brudzeń Landscape Park (=Brudzeński LP) by AbraszewskaKowalczyk et al. (2002) and 77 taxa of this family near Bromierzyk in the Kampinos National Park (=Kampinoski NP) by Szczepko et al. (2002). From the Załęcze Landscape Park (=Załęczański LP), located in the southwestern part of the Łódź Province, Kowalczyk \& 
Kurzac (2002) reported 90 species of Apidae. All those published data are not taken into account in the present report.

Scanty data on rare bees in central Poland can be found also in other works. Kowalczyk (1997) found Melecta luctuosa in Grzegorzowice in the Świętokrzyskie Mts. and Trachusa byssina in Gorzewo near Płock. Pesenko et al. (2000) reported on Evylaeus minutulus in Łódź, whereas W. Banaszak (2003) recorded Anthophora plagiata in Grzegorzowice.

The entomofauna of the Świętokrzyskie Mts. is relatively well studied thanks to the recent research conducted by Dylewska \& Bąk (2005), who listed 126 species of Apoidea, and by Kowalczyk \& Śliwiński (1988), who identified 16 bumblebee species in the Świętokrzyski NP. There are also several reports on the species composition of bumblebees and cuckoo bees of that area (Ćmak \& Szczypciak-Bąk 1987; Ruszkowski et al. 1989; Dylewska et al. 1998; Bąk 1998, 1999 a, b, 2003).

\section{MATERIAL AND METHODS}

This work is based mainly on material composed of 3887 bees caught in 1975-1992 by J. K. Kowalczyk and identified by J. Banaszak. Some specimens were kindly provided by other researchers. Their names are given in the list of localities and in Acknowledgements. We also used a small collection made during the $2^{\text {nd }}$ World War by E. Koeppen and F. Parrē. The bees come from the 125 localities listed below, lying mainly in central Poland (the northwestern part of the Małopolska Upland, the Łódź city and its environs, as well as the borderland of the Mazovian Lowland and the Wielkopolska-Kujawy Lowland), but some are situated in the Świętokrzyskie Mts. (Fig. 1).

Bees were collected systematically only in several localities throughout the growing season, i.e. from late March till early October (localities 4, 15, 47, 49, 64, 77, 101, 102, and 123). Data from other localities come mainly from summer months and are rather accidental.

Material was collected with a sweep net; no quantitative samples were taken. Bees were caught on flowers or near nests, in various, mainly open habitats. In the varied sites in the suburban zone of Łódź, these were mainly sand pits, mid-field ravines, afforested former farmland, birch woods, sides of unsurfaced roads between fields, ruderal habitats along railway tracks, neglected orchards, green areas around apartment blocks, and gardens near houses. Within the city, material was collected mainly in larger parks and in the Botanical Garden. Also the Łagiewniki Forest (Las Łagiewnicki), covering 1200 ha, was taken into account.

In rural areas, bees were caught mainly on wooden buildings and in former manor parks, on meadows, roadside willow trees, and wastelands. Less frequently samples were taken from grasslands on sand and limestone, xerothermic thickets, clayey river banks, quarries, loess ravines, and ruins of castles. In woodlands, open patches were preferred: openings, clearings, roadsides, forester's lodges, and forest edges.

Numbered localities were divided according to zoogeographic units. Description of each locality includes the UTM code, biotope type, collection year, and season (with the use of 3-letter abbreviations of months). Some localities are situated in protected areas: (1) national parks: Kampinos NP, Świętokrzyski NP; (2) nature reserves: 'Babsk', 'Konewka', 'Łaznów', 'Molenda', 'Popień', 'Spała', 'Wały', 'Wolbórka', ‘Żądłowice', 'Winnica', 'Kadzielnia', 'Wąwóz w Skałach'; or (3) landscape parks: Bolimów LP (=Bolimowski LP), Spała LP (=Spalski LP), Suchedniów-Oblęgorek LP (=Suchedniowsko-Oblęgorski LP), Sulejów LP (=Sulejowski LP), LP 'Międzyrzecza Warty i Widawki', and Łódź Hills LP (=LP ‘Wzniesień Łódzkich').

Relatively small numbers of data on bumblebees of the subgenus Terrestribombus Vogt are presented here because most of the material on this subgenus has already been published (Banaszak \& Rasmont 1994). 


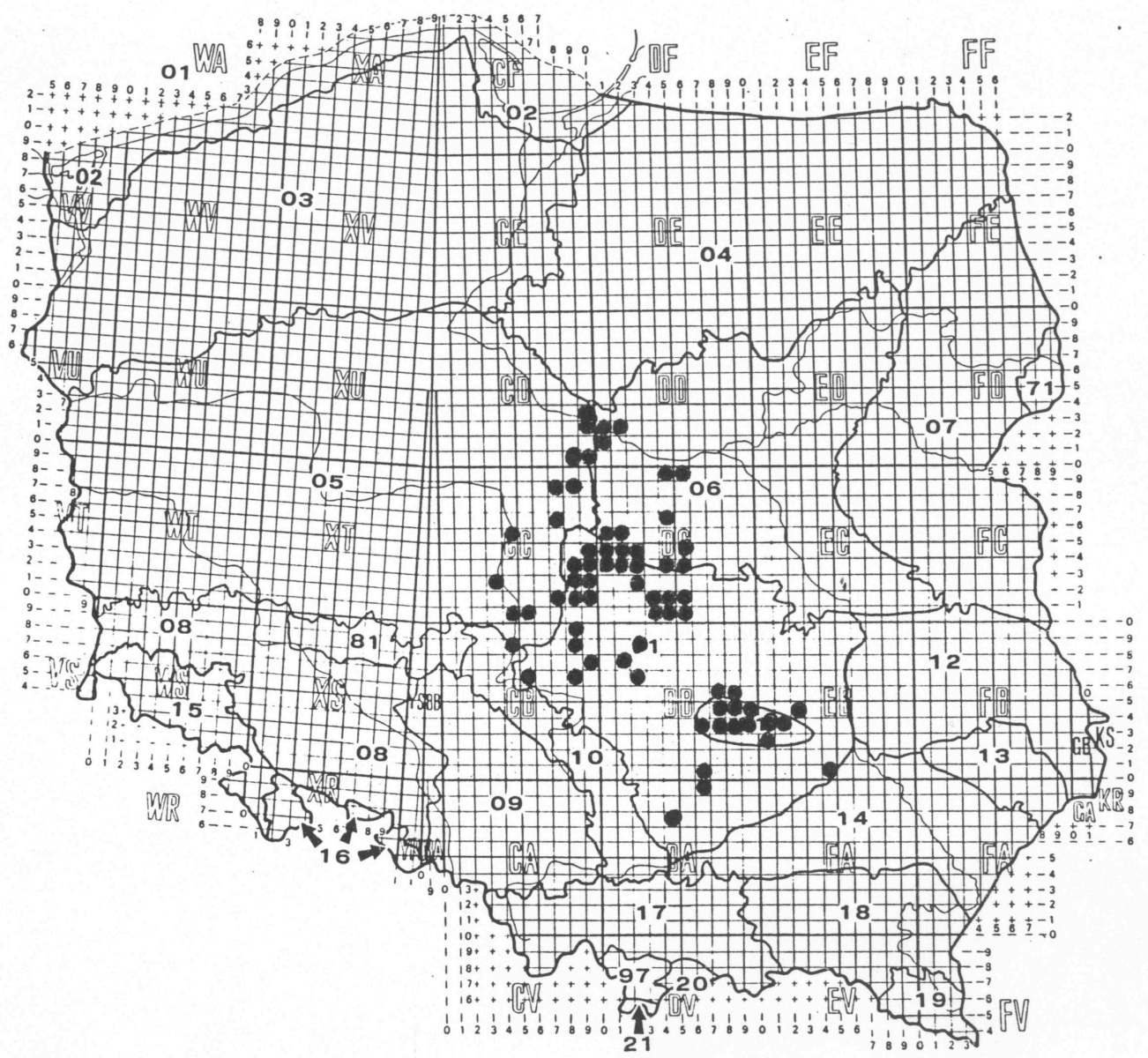

Fig. 1. Distribution of research sites.

\section{LIST OF LOCALITIES}

\section{Mazovian Lowland (1-17)}

1. 'Babsk' reserve (DC 54), typical oak-hornbeam forest with linden, 1988, leg. E. Szczepańska; (Jun-Sep).

2. Biskupice (DD 02), landing stage for yachts, old orchard, xerothermic thicket at oak forest edge, 1988; (Jun-Sep).

3. Bolimów (DC 49), meadows on the river Rawka, 1992; (May-Aug).

4. Bromierzyk, Kampinos NP (DC 59), former farmland, meadows on the river Łasica, 1987-1992; (May, Jul-Aug).

5. Budy Grabskie, Bolimów LP (DC 46), sandy grassland, 1995; (May).

6. Gorzewo (DD 01), sandy grassland, 1993; (Jul).

7. Grabie, Bolimów LP (DC 46), wooden forester's lodge, sandy grassland, 1993-1994; (Jun-Jul). Sep).

8. Marianów (CD 90), wooden buildings, pasture, 1988-1990, leg. J. Mańkowska (May- 
9. Murzynowo (CD 92), old orchard and wooden building near the Vistula, 1988; (Jun, Aug-Sep).

10. Osiny (CD 80), roadsides with rotten willow trees, 1990, leg. J. Mańkowska; (Aug).

11. Płock-Tum (DD 12), park, slope of the Vistula valley, 1987; 1994; (May-Jun).

12. Rawa Mazowiecka (DC 53), urban park, 1988, leg. E. Szczepańska; (Aug-Oct).

13. Sierakówek (CD 90), wooden buildings, sandy grassland, village park, 1990, leg. J. Mańkowska; (Jul-Aug).

14. Skierniewice-Rawka (DC 46), sandy grassland, 1993; (May).

15. Uniejewo (CD 93), slope of the Vistula valley, xerothermic grassland, 1988-1989, 1991, leg. M. Kobalczyk; (Jun-Sep).

16. Uroczysko Rawski Las (DC 43), old oak-pine forest, 1988, leg. E. Szczepańska; (Jul-Aug).

17. Ziemiary, Bolimów LP (DC 46), sandy grassland around forester's lodge, 1992-1993; (May, Aug).

\section{Małopolska Upland (18-88)}

18. Aleksandrów Łódzki (CC 82), 1990; (May).

19. Barycz on the Grabia (CC 71), dunes, sandy grassland, 1942, leg. E. Koeppen; 1943-1944, leg. F. Parrē; (May-Aug).

20. Bąkowa Góra, Sulejów LP (DB 26), tall herbs near ruins of a castle, 1992; (May).

21. Bełchatów (CB 89), slag heap near an opencast mine, 1986, leg. Z. Myślicka; (Jul).

22. Brzeziny (DC 14), sand pit, 1987; (Jul).

23. Budziszewice (DC 22), garden plots in oak-pine forest, 1994, leg. M. Mastalerz; (May).

24. Bukowiec, Łódź Hills LP (DC 04), moor, 1986; (Aug).

25. Chęciny (DB 63), xerothermic grassland near a castle, 1980; (Jul).

26. Dąbrowa, Łódź Hills LP (DC 04), sandy grassland, slopes of a ravine, 1986; (Jun-Sep).

27. Dąbrowa near Tomaszów Mazowiecki (DC 31), 1989, leg. J. Sobczak; (Aug).

28. Dąbrówka Duża, Łódź Hills LP (DC 14), rural habitats, 1976; (Jul).

29. Dobieszków, Łódź Hills LP (DC 04), roadsides with old poplars, side of a forest road, forest edge, 1986-1991; (Jun-Aug).

30. Dobryszyce (CB 86), rural habitats, 1988, leg. A. Zborowska; (Jul-Aug).

31. Gacki near Busk (DA 69), xerothermic grassland, 1977; (Jul).

32. Gałków (DC 13), mixed deciduous forest, 1987; 1990; (May-Jun).

33. Giełzów, Spała LP (DC 50), 1983; (Jul).

34. Glinnik (DC 05), 1984-1985, leg. J. Nadolski; (May-Jun).

35. Glinnik near Tomaszów Mazowiecki (DC 31), 1989, leg. J. Sobczak; (Sep).

36. Grocholice (CB 88), 1983, leg. Z. Myślicka; (Jul).

37. Grudzeń (DC 30), 1988, leg. E. Kwiatkowska; (Jul).

38. Inowłódz (DC 40), wooden cottage partly covered with clay, xerothermic grassland, 1983; (Jun).

39. Janinów, Łódź Hills LP (DC 14), beech forest, mixed deciduous forest, 1986-1991; (May-Sep).

40. Justynów (DC 03), sandy grassland, 1944, leg. F. Parrē; 1990; (May-Jul).

41. Kalonka, Łódź Hills LP (DC 04), rural habitats, 1986; (May-Aug).

42. Kolumna (CC 71), 1942, leg. E. Koeppen; (Jul).

43. 'Konewka' reserve, Spała LP (DC 41), xerothermic oak forest, 1980, leg. J. Kurzawa; 1988, leg. E. Kwiatkowska; (Apr, Jun-Aug).

44. Koprzywnica (EB 40), 1981; (May). 
45. Ludwików (DC 30), wooden buildings, 1988, leg. E. Kwiatkowska; (Jul-Aug).

46. 'Łaznów' reserve (DC 30), natural fir forest, 1991; (May).

47. Łódź (CC 83, CC 92, CC 94, DC 03), parks, ruderal habitats, 1976-1992; (Mar-Sep).

48. Łódź - Łagiewniki Forest, Łódź Hills LP (CC 94), mixed deciduous forest, 1984-1989; (Apr-Sep).

49. Łódź-Stoki (CC 93), green areas around apartment blocks, former manor park, rural habitats, sand pit, 1976-1992; (Apr-Sep).

50. Michałów (DC 24), sandy grassland on a hill on the river Mroga, 1974-1976; (Jul-Aug).

51. Modlica (CC 92), 1940, leg. E. Koeppen; 1943-1944, leg. F. Parrē; 1947, leg. Z. Śliwiński; 1980; (May-Aug).

52. Mogilno (CC 81), limestone quarry, 1990; (Jul).

53. 'Molenda' reserve (CC 91), beech-fir forest, 1980; (Jul).

54. Nagórzyce, Sulejów LP (DC 30), caves in chalk sandstone, 1983; (May).

55. Niecki, Łódź Hills LP (DC 04), rural habitats, 1986; (Jun, Aug).

56. Niesułków Kolonia, Łódź Hills LP (DC 15), pasture overgrown with broom (Cytisus scoparius) on a hill on the river Moszczenica, 1993-1994; (Jul-Aug).

57. Pińczów (DA 69), 1976, leg. M. Wanat; (Aug).

58. Podoba (DC 31), wooden building, 1988, leg. E. Kwiatkowska; (Aug).

59. Polichno (DA 69), xerothermic grassland, 1972, leg. A. Liana; (Jun).

60. 'Popień' reserve (DC 23), old pine forest on a fertile site, 1976-1977; 1991; (May-Jul).

61. Pytowice (CB 97), mixed deciduous forest, 1987, leg. Z. Myślicka; (May, Aug).

62. Racławice (DA 47), xerothermic grassland, 1985; (May).

63. Ręczno, Sulejów LP (DB 17), limestone quarry, 1985; (Jun).

64. Rogów (DC 24), wooden buildings, arboretum, 1975-1977; (Apr-Sep).

65. Rzgów (CC 92), old barn, 1980; (Jul).

66. Skowronno (DB 60), xerothermic grassland, 1972, leg. A. Liana; (Jun).

67. Słotwiny (DC 23), wooden buildings of a railway station and near railway tracks, 1992, leg. E. Ambrozik; (Jun-Jul).

68. Smardzew (CC 94), rural habitats, 1990; (May, Aug).

69 'Spała' reserve, Spała LP (DC 40), old oak-pine stand in oak-hornbeam forest, 1976, leg. B. Soszyński; (Jun).

70. Suchedniów (DB 85), mixed deciduous forest, 1986; (May).

71. Sulejów-Podklasztorze (DB 28), xerothermic grasslands and thickets, 1976, leg. B. Soszyński; (Jul).

72. Suchedniów-Oblęgorek LP (DB 75), meadow on the river Krasna, mixed deciduous forest, 1981; (Jul).

73. Szewna (EB 24), loess ravines, 1979; (Sep).

74. Szymaniszki, Łódź Hills LP (DC 14), pine-oak forest on slopes of the Moszczenica valley, 1987; (Jul).

75. Szynkielów, LP 'Międzyrzecza Warty i Widawki’ (CB 48), dune and mire, 1992; (Jun-Jul).

76. Tomaszów Mazowiecki - Białobrzegi (DC 31), stone barn, 1988-1989, leg. E. Kwiatkowska; (Jul-Sep).

77. Tomaszów Mazowiecki - Księża Góra, Spała LP (DC 31), sandy grassland, 1988-1989, leg. E. Kwiatkowska; (Jun-Sep).

78. Tuszyn-Poddębina (CC 91), mixed deciduous forest, 1947, leg. Z. Śliwiński; 1980; (Jul-Aug). 
79. 'Wały' reserve (DA 47), xerothermic grassland, 1985; (May).

80. Wagry (DC 23), rural habitats on the river Mroga, 1975-1976; (Jul-Sep).

81. Wiączyń (DC 03), wooden buildings and forest roads, 1982-1986; (May-Jul).

82. Wiśniowa Góra (DC 03), mixed deciduous forest, 1990; (Jun).

83. 'Wolbórka' reserve (CC 91), alder swamp forest, alluvial forest, 1980; (May, Jul).

84. Woźniki (CB 86), wooden buildings, 1989, leg. A. Zborowska; (Jul-Aug).

85. Wykno (DC 22), railway station, wooden building, 1992; (May).

86. Wymysłów (CC 82), 1984-1986, leg. C. Watała; (May).

87. Zalesiczki (CB 86), wooden building, 1989, leg. A. Zborowska; (May-Aug).

88. 'Żądłowice' reserve, Spała LP (DC 51), alder forest, oak-hornbeam forest, pine forest, 1983; (Jul).

\section{Wielkopolska-Kujawy Lowland (89-95)}

89. Burzenin, LP 'Międzyrzecza Warty i Widawki' (CC 50), xerothermic grasslands and thickets on the Vistula, 1973, 1977, leg. B. Soszyński; 1987; (Jul-Sep).

90. Byszew (CC 88), oak-hornbeam forest, 1988; (May).

91. Daszyna (CC 78), historical village park, wooden manor house, 1988; (May).

92. Łęczyca-Tum (CC 76), 1990; (Jun).

93. Małkow on the Warta (CC 32), ravines on the left bank of the river, 1984, leg. M. Wanat; (Jun).

94. Uniejów (CC 45), historical park around a castle, 1988; (Jun).

95. 'Winnica' reserve, LP 'Międzyrzecza Warty i Widawki' (CC 40), xerothermic thickets and gradually overgrown xerothermic grassland, 1992; (Jul).

\section{Wieluń-Kraków Upland (96)} (Aug).

96. Działoszyn (CB 56), xerothermic thickets and grasslands, 1976, leg. B. Soszyński;

\section{Świętokrzyskie Mountains (97-125)}

97. Bartoszowiny (EB 03), 1983; (Jul).

98. Belno (DB 85), oak forest edge, 1980; (Jul).

99. Bodzentyn (DB 94), wooden buildings, ruins of a castle, 1982; (Aug).

100. Celiny, Świętokrzyski NP (DB 93), 1982-1984; (Mar, Aug).

101. Chełmowa Góra, Świętokrzyski NP (EB 03), wooden buildings, meadows at forest edge, 1978-1985; (May-Aug).

102. Cząstków-Zapusty (EB 03), xerothermic thickets and grasslands, 1979, 1984; (Apr, Jul-Aug).

103. Dąbrowa (DB 93), 1980, leg. J. Szczypciak; (Jul).

104. Dębno (DB 93), roadsides, 1983; (Jul).

105. Drogosiowe (EB 02), wooden cottages, 1983; (Jul).

106. Góra Łysica, Świętokrzyski NP (DB 93), 1982; (Aug).

107. Góra Radostowa (DB 84), 1981, leg. A. Liana; (Jul).

108. Grzegorzowice (EB 13), mainly geological reserve 'Wąwóz w Skałach', 1979-1984; (May, Jul-Aug).

109. Huta Podłysica (DB 93), forest edge at the border of Świętokrzyski NP, 1983-1984; (Apr, Aug).

110. Jaworze (DB 74), old wooden cottages, 1981; (May).

111. 'Kadzielnia' reserve (DB 73), geological reserve in Kielce, former quarry, 1980; (May). 
112. Kakonin (DB 93), forest edge at the border of Świętokrzyski NP, 1982-1983; (Jul-Aug).

113. Kaniów (DB 75), 1980, leg. B. Gałczyńska; (Jul).

114. Krajno-Pogorzele (DB 83), rural habitats, 1982; (Jun, Aug).

115. Kobyla Góra (EB 03), 1983; 1987; (Jul-Aug).

116. Leszczyny (DB 83), water-gap of the river Lubrzanka, 1978; (May).

117. Nowa Słupia (EB 03), open-air ethnographic museum, grassland, thickets at the border of Świętokrzyski NP, 1979-1980; 1987; (Jun-Aug).

118. Pasmo Klonowskie (DB 84), 1982; (Apr).

119. Podlesie (DB 93), forest edge at the border of Świętokrzyski NP, 1982; (Sep).

120. Rudki (EB 03), ruderal vegetation of a former mine, 1984; (Aug).

121. Szklana Huta (EB 03), meadows at forest edge near the border of the Świętokrzyski NP, 1979-1984; (Jul-Aug).

122. Święta Katarzyna, Świętokrzyski NP (DB 93), near narrow-gauge railway tracks, 1982; 1985; (May-Jun, Aug-Sep).

123. Święty Krzyż, Świętokrzyski NP (EB 03), mainly the glade Bielnik with meadow dominated by Arrhenatherum elatioris, 1978-1992; (Mar-May, Jul-Sep).

124. Zachełmie (DB 84), 1980, leg. B. Gałczyńska; (Jul).

125. Zagnańsk (DB 74), 1980; (May, Jul).

\section{RESULTS}

The 3887 collected bees are members of 233 bee species (Table 1), which account for $49.1 \%$ of all species of Apiformes in Poland (Banaszak 2004). It is noteworthy that a large group of species was recorded on the Łódź Hills, lying at the border between the uplands of southern Poland and lowlands of northern Poland. For this reason it was rarely penetrated by entomologists in the old days. The study area (except the Świętokrzyskie Mts. in the eastern Małopolska Upland) is characterized by a relatively low geomorphological variation. In this study, 218 bee species were recorded in the northwestern part of the Małopolska Upland (mainly on the Łódź Hills), 89 in the Świętokrzyskie Mts., and 49 in the Vistula valley near Płock. These values are relatively low in comparison with earlier published data: 327 bee species in the Wielkopolska-Kujawy Lowland (Banaszak 1982), 274 in the Małopolska Upland (Loziński 1920), and 254 in the Mazovian Lowland (Banaszak \& Plewka 1981). From the Świętokrzyskie Mts., 126 species have been reported recently (Dylewska \& Bąk 2005). Contributions of individual subfamilies to the bee fauna of central Poland were similar to their contributions to the whole Polish bee fauna: Colletinae 9.4\% (in Poland 9.0\%), Andreninae 20.6\% (20.9\%); Halictinae 20.2\% (22.4\%), Melittinae 2.6\% (2.3\%), Megachlinae 20.6\% (18.8\%), Anthophorinae 15.0\% (18.1\%) and Apinae 11.6\% (8.5\%).

The most abundant species were Bombus lucorum $(4.2 \%$ of the total catch) and its cleptoparasite Psithyrus bohemicus (6.8\%). They were found mainly in the Świętokrzyskie Mts., where also other bumblebees and cuckoo bees are common: Bombus pascuorum $(3.7 \%$ of bees caught there), B. lapidarius (2.4\%) and Psithyrus campestris $(2.4 \%)$. In the area of Łódź Hills, the dominant species were Heriades truncorum (4.3\%) and Hylaeus communis (2.7\%). It is noteworthy that those species were not only most abundant, but also their localities were the most numerous. For example, Heriades truncorum was recorded in 31 localities, Hylaeus communis in 37, while Psithyrus bohemicus in 21 localities. Thus their largest contribution to the total catch is not a matter of chance or selective catching, but those species were truly common in the study area. It must be noted that in the Wielkopolska-Kujawy Lowland, 
Hylaeus communis and Heriades truncorum accounted for less than $1 \%$ each of the bee community. Both species were less numerous also in the Mazovian Lowland.

Rare species, represented in the analysed material by 1-3 individuals, accounted for $30.5 \%$ of the total number ( 71 out of 233 species). Their list, presented below, includes information on their localities, collection dates, and sex. Many of the rare species are found infrequently also in other parts of Poland. However, some are very rare, known only from single localities. The most interesting among them include:

Hylaeus gredleri - 6 individuals caught in Łódź or its vicinity; recorded in Poland only in the Kraków-Częstochowa Upland.

Andrena lapponica - species associated with forests, especially with Vaccinium species, so it is rare in the largely deforested study area.

A. mitis - very rare, found in Silesia, Kraków, and near Tarnów.

A. suerinensis - rare, sub-Mediterranean, scattered throughout Poland.

A. synadelpha - found mainly in West Europe, reaching to Poland and Turkey, rare in the Czech Republic and eastern Germany (Dylewska 2000, Osytshnjuk et al. 2005). In Poland reported from Opole (Dylewska 2000), but this needs to be confirmed.

Lasioglossum prasinum - known from single localities.

L. subfasciatum - known from several dozen localities in northwestern Poland.

Evylaeus limbellus - very rare, known in Poland from single localities, only one of them confirmed: Kazimierz Dolny.

E. semilucens - solitary, known in Poland from about a dozen localities.

E. tarsatus - rare throughout Poland, associated with steppes and sandy sites.

Osmia mustelina - poorly studied in Poland, rarely observed but in various regions.

O. uncinata - in Poland rather rare, although reported from various regions.

Megachile pilidens - known from some localities in the Sandomierz Lowland and Lower Silesia.

Anthophora plagiata - now very rare, although it was very common on clay walls of buildings in the 1970s in Polish lowlands. W. Banaszak (2005) found only one small local population of this species in the Wielkopolska-Kujawy Lowland, in Dziekanowice near Gniezno.

Table 1. List of species of Apiformes from Central Poland.

\begin{tabular}{|c|c|c|c|c|c|}
\hline No & Species & $\begin{array}{l}\text { Number } \\
\text { of } \\
\text { specimens } \\
\text { N/qq }\end{array}$ & $\begin{array}{l}\text { Mazovian } \\
\text { Lowland }\end{array}$ & $\begin{array}{l}\text { Małopolska } \\
\text { Upland }\end{array}$ & $\begin{array}{l}\text { Świętokrzyskie } \\
\text { Mountains }\end{array}$ \\
\hline 1 & 2 & 3 & 4 & 5 & 6 \\
\hline & Colletidae & & & & \\
\hline 1 & Hylaeus annularis (Kirby, 1802) & $7 / 5$ & $4,5,15$ & 26,30 & - \\
\hline 2 & Hylaeus bisinuatus Förster, 1871 & $4 / 3$ & 15 & 30,47 & - \\
\hline 3 & Hylaeus brevicornis Nylander, 1852 & $14 / 12$ & $8,12,13$ & $26,47,64,75$ & 102,108 \\
\hline 4 & Hylaeus communis Nylander, 1852 & $104 / 77$ & $\begin{array}{l}1,2,6,8,9, \\
11-13,15\end{array}$ & $\begin{array}{l}26,29,30,39 \\
43,47-49,53\end{array}$ & $101,102,108$ \\
\hline & & & & $\begin{array}{c}55,57,58,60, \\
64,69,72,76- \\
78,81,83,87\end{array}$ & \\
\hline 5 & Hylaeus confusus Nylander, 1852 & $9 / 7$ & $6,8,9,13$ & 43,56 & - \\
\hline 6 & Hylaeus difformis (Eversmann, 1852) & $10 / 8$ & 8,9 & $43,64,77$ & 102,114 \\
\hline 7 & Hylaeus gibbus Saunders, 1850 & $1 / 0$ & - & 56 & - \\
\hline 8 & $\begin{array}{l}\text { Hylaeus gracilicornis (F. Morawitz, } \\
1867 \text { ) }\end{array}$ & $1 / 1$ & - & - & 102 \\
\hline 9 & Hylaeus gredleri Förster, 1871 & $6 / 4$ & - & $47,49,57,64$ & - \\
\hline
\end{tabular}


Apiformes of central Poland

\begin{tabular}{|c|c|c|c|c|c|}
\hline 1 & 2 & 3 & 4 & 5 & 6 \\
\hline 10 & Hylaeus hyalinatus Smith 1842 & $15 / 11$ & 7,8 & $47,49,60,77$ & - \\
\hline 11 & Hylaeus nigritus (Fabricius, 1758) & $4 / 0$ & - & 47,75 & 123 \\
\hline 12 & Hylaeus pictipes Nylander, 1852 & $3 / 2$ & 8 & 49,83 & - \\
\hline 13 & Hylaeus punctatus (Brullē, 1832) & $1 / 1$ & - & 47 & - \\
\hline 14 & Hylaeus punctulatissimus Smith, 1842 & $1 / 1$ & - & 64 & - \\
\hline 15 & Hylaeus rinki (Gorski, 1852) & $1 / 1$ & - & 77 & - \\
\hline 16 & Hylaeus signatus (Panzer, 1798) & $20 / 16$ & - & $47,75,76$ & 123 \\
\hline 17 & Hyaleus sinuatus (Schenck, 1853) & $33 / 21$ & 7,13 & $\begin{array}{c}30,60,64,71 \\
76-78,87\end{array}$ & $\begin{array}{c}101,102,108, \\
112,114\end{array}$ \\
\hline 18 & Colletes cunicularius (Linnaeus, 1761) & $10 / 6$ & 4 & $47,49,83$ & - \\
\hline 19 & Colletes daviesanus Smith, 1846 & $40 / 30$ & $8,9,15$ & $\begin{array}{c}37,45,47,49 \\
76,77\end{array}$ & $108,112,120$ \\
\hline 20 & Colletes fodiens (Fourcroy, 1785) & $1 / 0$ & - & 49 & - \\
\hline 21 & Colletes similis Schenck, 1853 & $4 / 4$ & 1,4 & 40,48 & - \\
\hline 22 & Colletes succinctus (Linnaeus, 1785) & $3 / 3$ & - & 24,49 & - \\
\hline & Andrenidae & & & & \\
\hline 23 & Andrena alfkenella Perkins, 1914 & $1 / 0$ & - & 49 & - \\
\hline 24 & Andrena apicata Smith, 1847 & $2 / 2$ & - & 61,83 & - \\
\hline 25 & Andrena barbilabris (Kirby, 1802) & $4 / 2$ & - & $48,49,125$ & \\
\hline 26 & Andrena bicolor Fabricius, 1775 & $11 / 8$ & - & 47 & 123 \\
\hline 27 & Andrena bimaculata (Kirby, 1802) & $11 / 4$ & - & 47,49 & - \\
\hline 28 & Andrena chrysopyga Schenck, 1853 & $5 / 3$ & - & 26,49 & - \\
\hline 29 & Andrena chrysosceles (Kirby, 1802) & $2 / 2$ & - & 47 & - \\
\hline 30 & Andrena cineraria (Linnaeus, 1758) & $8 / 6$ & - & $41,47-49,60,83$ & - \\
\hline 31 & Andrena clarkella (Kirby, 1802) & $26 / 16$ & - & $43,47-49,70$ & $\begin{array}{c}100,109,118, \\
123\end{array}$ \\
\hline 32 & Andrena denticulata (Kirby, 1802) & $10 / 4$ & - & 48,49 & 101,108 \\
\hline 33 & Andrena dorsata (Kirby, 1802) & $32 / 20$ & 8 & $\begin{array}{c}30,36,47,49 \\
81,83\end{array}$ & 101,123 \\
\hline 34 & Andrena flavipes Panzer, 1799 & $35 / 12$ & 10,15 & $21,47,49,76,77$ & 111 \\
\hline 35 & Andrena fucata Smith, 1847 & $3 / 2$ & - & 48,53 & - \\
\hline 36 & Andrena fulva (Müller, 1766) & $48 / 40$ & 11 & $41,47-49,81$ & - \\
\hline 37 & Andrena fuscipes (Kirby, 1802) & $5 / 4$ & 17 & 24,41 & - \\
\hline 38 & Andrena gelriae Van der Vecht, 1927 & $4 / 1$ & - & $26,32,47$ & - \\
\hline 39 & Andrena gravida Imhoff, 1832 & $9 / 9$ & - & $47,62,76$ & - \\
\hline 40 & Andrena haemorrhoa (Fabricius, 1781) & $55 / 18$ & 8 & $\begin{array}{c}47-49,51,62 \\
64,68,83\end{array}$ & $109,122,123$ \\
\hline 41 & Andrena hattorfiana (Fabricius, 1775) & $16 / 14$ & - & $19,41,47,52,77$ & $108,113,117$ \\
\hline 42 & Andrena helvola (Linnaeus, 1758) & $3 / 2$ & - & $48,49,81$ & - \\
\hline 43 & Andrena humilis Imhoff, 1832 & $3 / 3$ & - & 41,51 & 101 \\
\hline 44 & Andrena jakobi Perkins, 1931 & $35 / 31$ & 5 & $41,47,49,68$ & 123 \\
\hline 45 & Andrena labialis (Kirby, 1802) & $1 / 0$ & 15 & - & - \\
\hline 46 & Andrena labiata Fabricius, 1781 & $11 / 4$ & $4,14,17$ & $32,47,48$ & - \\
\hline 47 & Andrena lapponica Zetterstedt, 1838 & $1 / 1$ & - & 46 & - \\
\hline 48 & Andrena marginata Fabricius, 1776 & $2 / 2$ & - & 57 & - \\
\hline 49 & Andrena minutula (Kirby, 1802) & $6 / 6$ & - & $47,49,83$ & 123 \\
\hline 50 & Andrena minutuloides Perkins, 1914 & $12 / 12$ & - & 47,49 & 108 \\
\hline 51 & Andrena mitis Schmiedeknecht, 1883 & $1 / 1$ & - & 47 & - \\
\hline 52 & Andrena nigroaenea (Kirby, 1802) & $16 / 8$ & $4,5,11$ & $39,47,49,83$ & 108 \\
\hline 53 & Andrena nitida (Müller, 1776) & $26 / 16$ & 11 & $47,49,61$ & $101,102,123$ \\
\hline 54 & Andrena nitidiuscula Schenck, 1853 & $1 / 1$ & - & - & 102 \\
\hline 55 & Andrena ovatula (Kirby, 1802) & $3 / 2$ & - & 48,49 & 117 \\
\hline 56 & Andrena pilipes (Fabricius, 1871) & $36 / 12$ & 8 & $\begin{array}{c}29,39-41,47,49 \\
64,88\end{array}$ & - \\
\hline 57 & Andrena praecox (Scopoli, 1763) & $19 / 17$ & - & $23,47-49$ & 123 \\
\hline 58 & Andrena proxima (Kirby, 1802) & $5 / 5$ & 2 & 47 & 101,123 \\
\hline 59 & Andrena rosae Panzer, 1801 & $2 / 1$ & - & - & 102 \\
\hline 60 & Andrena ruficrus Nylander, 1848 & $2 / 2$ & - & 51 & - \\
\hline 61 & Andrena schencki F. Morawitz, 1866 & $1 / 0$ & - & 51 & - \\
\hline
\end{tabular}




\begin{tabular}{|c|c|c|c|c|c|}
\hline 1 & 2 & 3 & 4 & 5 & 6 \\
\hline 62 & Andrena subopaca Nylander, 1848 & $12 / 1$ & - & $47,49,78$ & 102,123 \\
\hline 63 & Andrena suerinensis Friese, 1884 & $1 / 0$ & - & 49 & - \\
\hline 64 & Andrena synadelpha Perkins, 1914 & $2 / 2$ & - & 49,68 & - \\
\hline 65 & Andrena tibialis (Kirby, 1802) & $10 / 7$ & 11 & 47,49 & - \\
\hline 66 & Andrena varians (Rossi, 1791) & $11 / 11$ & - & $41,47,49$ & 125 \\
\hline 67 & Andrena vaga Panzer, 1799 & $1 / 0$ & 4 & - & - \\
\hline 68 & Andrena ventralis Imhoff, 1832 & $3 / 3$ & - & 47,48 & - \\
\hline 69 & Andrena wilkella (Kirby, 1802) & $2 / 2$ & 8 & 26 & - \\
\hline 70 & Panurgus calcaratus (Scopoli, 1763) & $14 / 5$ & - & $19,47-49,53,64$ & - \\
\hline & Halictidae & & & & \\
\hline 71 & Halictus maculatus Smith, 1848 & $11 / 9$ & - & $48,49,55,62,77$ & 102,120 \\
\hline 72 & Halictus quadricinctus (Fabricius, 1775) & $10 / 5$ & - & $28,47,49-51$ & - \\
\hline 73 & Halictus rubicundus (Christ, 1791) & $24 / 18$ & 1,16 & $\begin{array}{l}26,39,41,47- \\
\quad 49,62,83\end{array}$ & - \\
\hline 74 & Halictus sexcinctus (Fabricius, 1775) & $18 / 6$ & $4,8,10,13$ & $\begin{array}{l}30,47,49-51 \\
\quad 68,75\end{array}$ & - \\
\hline 75 & Seladonia confusa (Smith, 1853) & $3 / 1$ & - & 19,30 & - \\
\hline 76 & Seladonia leucahenea (Ebmer, 1972) & $5 / 3$ & - & 39,49 & - \\
\hline 77 & Seladonia tumulorum (Linnaeus, 1758) & $70 / 63$ & $\begin{array}{l}1,2,4,8 \\
12,15,16\end{array}$ & $24,27,49,77$ & - \\
\hline 78 & Lasioglossum lativentre (Schenck, 1853) & $3 / 1$ & $1,2,12$ & - & - \\
\hline 79 & $\begin{array}{l}\text { Lasioglossum leucozonium (Schrank, } \\
\text { 1781) }\end{array}$ & $12 / 5$ & 1,13 & $\begin{array}{l}19,26,30,47 \\
49,76,77,81\end{array}$ & - \\
\hline 80 & Lasioglossum prasinum (Smith, 1848) & $1 / 1$ & 10 & - & - \\
\hline 81 & $\begin{array}{l}\text { Lasioglossum quadrinotatum (Kirby, } \\
\text { 1802) }\end{array}$ & $5 / 1$ & 12 & $30,39,47,49$ & - \\
\hline 82 & Lasioglossum sexnotatum (Kirby, 1802) & $26 / 26$ & 2,9 & $\begin{array}{r}26,35,39,41 \\
47-49,51,64,81\end{array}$ & $102,121,123$ \\
\hline 83 & $\begin{array}{l}\text { Lasioglossum subfasciatum (Imhoff, } \\
\text { 1832) }\end{array}$ & $3 / 2$ & - & 49 & 123 \\
\hline 84 & Lasioglossum xanthopus (Kirby, 1802) & $3 / 3$ & - & 49,66 & - \\
\hline 85 & Lasioglossum zonulum (Smith, 1848) & $4 / 4$ & 1,10 & 24,27 & - \\
\hline 86 & Evylaeus albipes (Fabricius, 1781) & $16 / 10$ & $1,2,9,16$ & $30,51,77,86$ & 101,123 \\
\hline 87 & Evylaeus calceatus (Scopoli, 1763) & $25 / 9$ & $\begin{array}{c}1,8-10,12 \\
13,16\end{array}$ & $27,35,47,48$ & - \\
\hline 88 & Evylaeus fulvicornis (Kirby, 1802) & $12 / 3$ & 2,9 & - & $\begin{array}{l}101,102,108, \\
109,123\end{array}$ \\
\hline 89 & Evylaeus laticeps (Schenck, 1869) & $8 / 2$ & - & 31,49 & - \\
\hline 90 & Evylaeus limbellus (F. Morawitz, 1876) & $1 / 1$ & 15 & - & - \\
\hline 91 & Evylaeus lucidulus (Schenck, 1861) & $2 / 1$ & 15 & 47 & - \\
\hline 92 & Evylaeus minutissimus (Kirby, 1802) & $1 / 0$ & 16 & - & - \\
\hline 93 & Evylaeus morio (Fabricius, 1793) & $20 / 5$ & $2,8,12,13$ & 48,77 & - \\
\hline 94 & Evylaeus parvulus (Schenck, 1853) & $6 / 3$ & $2,10,15$ & - & - \\
\hline 95 & Evylaeus pauxillus (Schenck, 1853) & $21 / 14$ & 1 & $47-49,77$ & 102 \\
\hline 96 & $\begin{array}{l}\text { Evylaeus quadrinotatulus (Schenck, } \\
1861 \text { ) }\end{array}$ & $10 / 4$ & $2,3,15$ & - & - \\
\hline 97 & Evylaeus rufitarsis (Zetterstedt, 1838) & $1 / 1$ & 15 & - & - \\
\hline 98 & Evylaeus semilucens (Alfken, 1914) & $2 / 0$ & - & 48 & - \\
\hline 99 & Evylaeus sexstrigatus (Schenck, 1869) & $18 / 14$ & $1,2,12,15$ & 47,49 & - \\
\hline 100 & Evylaeus tarsatus (Schenck, 1869) & $2 / 2$ & 1 & - & - \\
\hline 101 & Evylaeus villosulus (Kirby, 1802) & $1 / 1$ & - & 77 & - \\
\hline 102 & Systropha curvicornis (Scopoli, 1770) & $7 / 0$ & - & 19,40 & - \\
\hline 103 & Rophites quinquespinosus Spinola, 1808 & $3 / 2$ & - & 49,66 & - \\
\hline 104 & Sphecodes albilabris (Fabricius, 1793) & $15 / 3$ & 4,15 & 76,77 & - \\
\hline 105 & Sphecodes crassus Thomson, 1870 & $23 / 18$ & 2,15 & $27,30,49,77,78$ & 102,108 \\
\hline 106 & Sphecodes ephippius (Linnaeus, 1767) & $34 / 16$ & $2-4,15$ & $\begin{array}{c}26,47-49,64 \\
77,78\end{array}$ & 123 \\
\hline 107 & Sphecodes ferruginatus Hagens, 1882 & $3 / 3$ & - & 47,49 & - \\
\hline
\end{tabular}




\begin{tabular}{|c|c|c|c|c|c|}
\hline 1 & 2 & 3 & 4 & 5 & 6 \\
\hline 108 & Sphecodes geoffrellus (Kirby, 1802) & $12 / 9$ & 15 & $\begin{array}{c}49,57,59,68 \\
76,87\end{array}$ & 108 \\
\hline 109 & Sphecodes gibbus (Linnaeus, 1758) & $45 / 27$ & $2,8,13,15$ & $\begin{array}{c}19,28,32,39 \\
41,47,49,50, \\
71,73,77,80,83\end{array}$ & - \\
\hline 110 & Sphecodes longulus Hagens, 1882 & $14 / 4$ & 15 & $\begin{array}{c}26,30,47-49 \\
73,76\end{array}$ & 108,123 \\
\hline 111 & Sphecodes marginatus Hagens, 1882 & $1 / 1$ & - & 77 & - \\
\hline 112 & Sphecodes miniatus Hagens, 1882 & $10 / 2$ & 2,15 & $38,49,76$ & 108 \\
\hline 113 & Sphecodes monilicornis (Kirby, 1802) & $33 / 23$ & $4,8,13$ & $\begin{array}{c}19,29,43,49 \\
50,57,64,76, \\
77,86\end{array}$ & 102,105 \\
\hline 114 & Sphecodes niger Hagens, 1874 & $4 / 0$ & $1,2,4$ & 48 & - \\
\hline 115 & Sphecodes pellucidus Smith, 1845 & $25 / 24$ & $2,4,15$ & $34,47,49,86$ & - \\
\hline 116 & Sphecodes puncticeps Thomson, 1870 & $1 / 0$ & - & 49 & - \\
\hline 117 & Sphecodes reticulatus Thomson, 1870 & $12 / 8$ & 8 & $\begin{array}{l}26,30,38,47 \\
49,76,77\end{array}$ & - \\
\hline & Melittidae & & & & \\
\hline 118 & Dasypoda hirtipes (Harris, 1780) & $25 / 10$ & $1,12,13$ & $41,48-51$ & - \\
\hline 119 & Macropis europaea Warncke, 1973 & $10 / 6$ & 4 & $21,48,61,74$ & 102,121 \\
\hline 120 & Macropis fulvipes (Fabricius, 1804) & $14 / 4$ & 4 & $\begin{array}{c}32,47,48,72 \\
74,81\end{array}$ & 102,115 \\
\hline 121 & $\begin{array}{l}\text { Melitta haemorrhoidalis (Fabricius, } \\
1775)\end{array}$ & $1 / 1$ & - & & 108 \\
\hline 122 & Melitta leporina (Panzer, 1799) & $13 / 4$ & - & $19,39,47-49,78$ & - \\
\hline 123 & Melitta nigricans Alfken, 1905 & $5 / 4$ & $\begin{array}{c}3,4 \\
-\end{array}$ & 75 & - \\
\hline 124 & Anthidium manicatum (Linnaeus, 1758) & $19 / 8$ & - & $47,49,64,71,73$ & 102 \\
\hline 125 & $\begin{array}{l}\text { Proanthidium oblongatum Latreille, } \\
1809\end{array}$ & $6 / 3$ & - & $47,67,75$ & - \\
\hline 126 & Anthidiellum strigatum (Panzer, 1805) & $20 / 6$ & 4 & $\begin{array}{c}19,22,26,39 \\
40,47-49,64,75\end{array}$ & - \\
\hline 127 & Stelis breviuscula (Nylander, 1848) & $32 / 20$ & $2,8,9,13$ & $\begin{array}{l}29,30,37,45 \\
65,76-78,87\end{array}$ & - \\
\hline 128 & $\begin{array}{l}\text { Stelis minuta Lepeletier and Serville, } \\
1825\end{array}$ & $1 / 1$ & - & 29 & - \\
\hline 129 & Stelis ornatula (Klug, 1807) & $1 / 0$ & - & 47 & - \\
\hline 130 & Stelis phaeoptera (Kirby, 1802) & $9 / 9$ & 9 & $38,47,51,87$ & - \\
\hline 131 & Stelis punctulatissima (Kirby, 1802) & $5 / 4$ & 9 & 47,84 & 101 \\
\hline 132 & Stelis signata (Latreille, 1809) & $3 / 1$ & - & 64,75 & - \\
\hline 133 & Heriades crenulatus Nylander, 1856 & $10 / 8$ & 9 & $45,76,77$ & - \\
\hline 134 & Heriades truncorum (Linnaeus, 1758) & $168 / 108$ & $\begin{array}{l}1,4,8,9 \\
12,13,16\end{array}$ & $\begin{array}{c}19,30,35-38 \\
43,45,47-49 \\
58,64,72,76- \\
78,84,87\end{array}$ & $\begin{array}{c}101,105,108 \\
123\end{array}$ \\
\hline 135 & $\begin{array}{l}\text { Chelostoma campanularum (Kirby, } \\
\text { 1802) }\end{array}$ & $35 / 25$ & $8,9,16$ & $\begin{array}{c}26,27,30,49 \\
65,87\end{array}$ & 101,102 \\
\hline 136 & Chelostoma distinctum Stoeckhert, 1929 & $1 / 1$ & 8 & - & - \\
\hline 137 & Chelostoma florisomne (Linnaeus, 1758) & $35 / 19$ & 8 & $29,38,41,47,81$ & $98,110,123$ \\
\hline 138 & Chelostoma rapunculi (Lepeletier, 1841) & $50 / 23$ & $2,3,8$ & $37,47,49,72,87$ & $101,102,114$ \\
\hline 139 & $\begin{array}{l}\text { Anthocopa bidentata (F. Morawitz, } \\
\text { 1876) }\end{array}$ & $16 / 15$ & - & $58,76,77$ & - \\
\hline 140 & Hoplitis adunca (Panzer, 1798) & $10 / 5$ & 9 & $38,67,75$ & 117,120 \\
\hline 141 & Hoplitis anthocopoides (Schenck, 1853) & $1 / 1$ & - & 75 & - \\
\hline 142 & Hoplitis claviventris (Thomson, 1872) & $14 / 10$ & 4,15 & $32,47,48,63,75$ & - \\
\hline 143 & Hoplitis leucomelana (Kirby, 1802) & $2 / 2$ & - & 30,49 & - \\
\hline 144 & Osmia aurulenta (Panzer, 1799) & $4 / 3$ & - & 79 & - \\
\hline 145 & Osmia brevicornis (Fabricius, 1798) & $26 / 2$ & 8 & 47,85 & - \\
\hline
\end{tabular}




\begin{tabular}{|c|c|c|c|c|c|}
\hline 1 & 2 & 3 & 4 & 5 & 6 \\
\hline 146 & Osmia coerulescens (Linnaeus, 1758) & $16 / 12$ & 8 & $19,47,49$ & 101 \\
\hline 147 & Osmia fulviventris (Panzer, 1798) & $10 / 6$ & - & $38,72,81$ & 108,110 \\
\hline 148 & Osmia leaiana (Kirby, 1802) & $3 / 2$ & - & 32,78 & 108 \\
\hline 149 & Osmia mustelina Gerstaecker, 1841 & $3 / 2$ & - & 47,49 & - \\
\hline 150 & Osmia rufa (Linnaeus, 1758) & $41 / 17$ & 11 & $\begin{array}{l}19,40,47-49 \\
51,61,81\end{array}$ & 123 \\
\hline 151 & Osmia uncinata Gerstaecker, 1869 & $1 / 1$ & - & 68 & - \\
\hline 152 & $\begin{array}{l}\text { Chalicodoma ericetorum (Lepeletier, } \\
\text { 1841) }\end{array}$ & $16 / 2$ & - & $19,47,49,76$ & - \\
\hline 153 & Megachile alpicola Alfken, 1924 & $12 / 8$ & $2,4,14$ & $29,47,49$ & - \\
\hline 154 & $\begin{array}{l}\text { Megachile centuncularis (Linnaeus, } \\
1758 \text { ) }\end{array}$ & $4 / 3$ & 4 & 47,87 & 117 \\
\hline 155 & Megachile circumcincta (Kirby, 1802) & $19 / 11$ & 4,15 & $19,47-49,54,63$ & - \\
\hline 156 & Megachile leachella Curtis, 1828 & $11 / 7$ & 4 & 75 & - \\
\hline 157 & Megachile maritima (Kirby, 1802) & $10 / 8$ & 4,15 & $49,50,67,75$ & - \\
\hline 158 & Megachile pilidens Alfken, 1923 & $3 / 1$ & 4 & 47,75 & - \\
\hline 159 & Megachile rotundata (Fabricius, 1784) & $10 / 4$ & $1,4,8,15$ & 30 & - \\
\hline 160 & Megachile versicolor Smith, 1844 & $5 / 2$ & 4 & $29,30,48,49$ & - \\
\hline 161 & Megachile willughbiella (Kirby, 1802) & $19 / 3$ & 1 & $\begin{array}{l}25,32,43,47 \\
\quad 49,51,75\end{array}$ & 123 \\
\hline 162 & Coelioxys afra Lepeletier, 1841 & $4 / 4$ & 3 & 75,77 & - \\
\hline 163 & Coelioxys aurolimbata Förster, 1853 & $1 / 0$ & - & 47 & - \\
\hline 164 & Coelioxys brevis Eversmann, 1852 & $5 / 3$ & 4 & 19,50 & - \\
\hline 165 & Coelioxys conoidea (Illiger, 1806) & $8 / 5$ & 4 & $19,51,75$ & - \\
\hline 166 & Coelioxys elongata Lepeletier, 1841 & $2 / 1$ & 4 & 49 & - \\
\hline 167 & Coelioxys inermis (Kirby, 1802) & $1 / 1$ & - & 49 & - \\
\hline 168 & Coelioxys mandibularis Nylander, 1848 & $4 / 3$ & - & 47 & - \\
\hline 169 & $\begin{array}{l}\text { Coelioxys quadridentata (Linnaeus, } \\
1758 \text { ) }\end{array}$ & $8 / 7$ & 4,15 & $40,49,75,77,78$ & - \\
\hline 170 & Coelioxys rufescens Lepeletier, 1825 & $7 / 4$ & - & $29,30,49,64$ & 123 \\
\hline 171 & Coelioxys rufocaudata Smith, 1854 & $2 / 1$ & - & 60,77 & - \\
\hline & Apidae & & & & \\
\hline 172 & Nomada femoralis F. Morawitz, 1869 & $3 / 3$ & - & 49 & - \\
\hline 173 & Nomada flava Panzer, 1798 & $9 / 4$ & - & 47,49 & 111,116 \\
\hline 174 & Nomada flavoguttata (Kirby, 1802) & $14 / 12$ & - & $\begin{array}{c}29,32,47-49 \\
60,64\end{array}$ & 111 \\
\hline 175 & Nomada flavopicta (Kirby, 1802) & $19 / 12$ & - & $\begin{array}{c}19,40,47,49 \\
57,77\end{array}$ & 102,120 \\
\hline 176 & Nomada fulvicornis Fabricius, 1793 & $48 / 27$ & 11 & $\begin{array}{c}29,35,41,47 \\
49,51,53,78\end{array}$ & 111,121 \\
\hline 177 & Nomada fuscicornis Nylander, 1848 & $3 / 2$ & - & 48,49 & - \\
\hline 178 & Nomada goodeniana (Kirby, 1802) & $13 / 13$ & 15 & $47,49,51,83,86$ & 121 \\
\hline 179 & Nomada lathburiana (Kirby, 1802) & $6 / 6$ & - & $47,49,83$ & - \\
\hline 180 & Nomada marshamella (Kirby, 1802) & $17 / 14$ & - & $47,49,51,64$ & $109,116,121$ \\
\hline 181 & Nomada moeschleri Alfken, 1913 & $34 / 28$ & 3 & $\begin{array}{c}32,48,49,60 \\
64,70,81,82,86\end{array}$ & 123 \\
\hline 182 & Nomada ochrostoma Zetterstedt, 1838 & $9 / 7$ & 3,4 & 49 & - \\
\hline 183 & Nomada panzeri Lepeletier, 1841 & $2 / 1$ & - & 49 & - \\
\hline 184 & Nomada roberjeotiana Panzer, 1799 & $36 / 19$ & 8 & $\begin{array}{c}19,26,28,33 \\
47,49,53,77 \\
78,80,83\end{array}$ & 102 \\
\hline 185 & Nomada ruficornis (Linnaeus, 1758) & $38 / 33$ & 15 & $\begin{array}{c}48,49,61,64, \\
70,77,78,80, \\
\quad 81,86\end{array}$ & $\begin{array}{c}101,109,121, \\
122\end{array}$ \\
\hline 186 & Nomada rufipes Fabricius, 1793 & $8 / 7$ & 4 & 24,64 & - \\
\hline 187 & Nomada sexfasciata Panzer, 1799 & $3 / 3$ & & $34,50,64$ & - \\
\hline 188 & Nomada sheppardana (Kirby, 1802) & $5 / 5$ & - & $48,49,81$ & 102 \\
\hline 189 & Nomada signata Jurine, 1807 & $15 / 7$ & - & $32,34,48,49$ & 111,123 \\
\hline 190 & Nomada zonata Panzer, 1798 & $4 / 1$ & - & 49 & - \\
\hline
\end{tabular}




\begin{tabular}{|c|c|c|c|c|c|}
\hline 1 & 2 & 3 & 4 & 5 & 6 \\
\hline 191 & Anthophora bimaculata (Panzer, 1798) & $7 / 3$ & - & $\begin{array}{c}19,33,49,50 \\
67,76\end{array}$ & - \\
\hline 192 & Anthophora furcata (Panzer, 1798) & $20 / 6$ & 9 & $29,47,49,53,74$ & 102,123 \\
\hline 193 & Anthophora plagiata (Illiger, 1806) & $1 / 0$ & - & 47 & - \\
\hline 194 & Anthophora plumipes (Pallas, 1772) & $23 / 10$ & 11 & $\begin{array}{l}18,20,44,47 \\
48,62\end{array}$ & 123 \\
\hline 195 & $\begin{array}{l}\text { Anthophora quadrimaculata (Panzer, } \\
\text { 1806) }\end{array}$ & $18 / 11$ & - & $47,49,76,77$ & 112 \\
\hline 196 & Anthophora retusa (Linnaeus, 1758) & $3 / 1$ & - & 49 & - \\
\hline 197 & Biastes emarginatus (Schenck, 1853) & $1 / 1$ & - & 49 & - \\
\hline 198 & Ceratina cyanea (Kirby, 1802) & $16 / 13$ & 4,8 & $26,47,49,64,77$ & - \\
\hline 199 & Epeoloides coecutiens (Fabricius, 1775) & $2 / 0$ & - & 75 & 107 \\
\hline 200 & Epeolus cruciger (Panzer, 1799) & $4 / 3$ & - & 19,47 & - \\
\hline 201 & Epeolus variegatus (Linnaeus, 1758) & $22 / 16$ & 2,15 & $41,47-49,75$ & - \\
\hline 202 & Eucera longicornis (Linnaeus, 1758) & $9 / 2$ & - & $47,49,51$ & - \\
\hline 203 & Melecta albifrons (Förster, 1771) & $7 / 3$ & 11 & $47,49,62,64$ & - \\
\hline 204 & Tetralonia dentata (Klug, 1835) & $9 / 3$ & - & $42,50,80$ & - \\
\hline 205 & Thyreus histrionicus (Illiger, 1806) & $3 / 2$ & - & 40,50 & - \\
\hline 206 & Thyreus orbatus (Lepeletier, 1841) & $3 / 1$ & 4 & 45,49 & - \\
\hline 207 & Bombus cryptarum (Fabricius, 1775) & 21 & & & \\
\hline 208 & $\begin{array}{l}\text { Bombus distinguendus (F. Morawitz, } \\
1869 \text { ) }\end{array}$ & $2 / 1$ & - & 32 & - \\
\hline 209 & Bombus hortorum (Linnaeus, 1761) & $69 / 16$ & 4 & $\begin{array}{l}30,35,47-49 \\
51,55,72\end{array}$ & $\begin{array}{l}101,102,109 \\
114,121-125\end{array}$ \\
\hline 210 & Bombus humilis Illiger, 1806 & $3 / 3$ & - & 73 & 102 \\
\hline 211 & Bombus hypnorum (Linnaeus, 1758) & $40 / 13$ & - & $47-49,51,76,77$ & $\begin{array}{c}101,102,106, \\
109,112,113, \\
121,125\end{array}$ \\
\hline 212 & Bombus jonellus (Kirby, 1802) & $5 / 0$ & 4 & 48,76 & - \\
\hline 213 & Bombus lapidarius (Linnaeus, 1758) & $94 / 33$ & $\begin{array}{c}1,4,8,12 \\
15\end{array}$ & $\begin{array}{c}24,30,47,49 \\
51,58,61,76-78\end{array}$ & $\begin{array}{c}99,100-102, \\
114,117,121- \\
123\end{array}$ \\
\hline 214 & Bombus lucorum (Linnaeus, 1761) & $159 *$ & $4,8,9,12$ & 48 & - \\
\hline 215 & Bombus magnus Vogt, 1911 & 1 & & & \\
\hline 216 & Bombus muscorum (Linnaeus, 1758) & $21 / 7$ & 4,8 & $19,24,26,48,51$ & 114 \\
\hline 217 & Bombus pascuorum (Scopoli, 1763) & $145 / 71$ & 4,8 & $\begin{array}{c}26,30,35,39 \\
41,43,47-49 \\
51,58,72,77,78\end{array}$ & $\begin{array}{c}99-103,106, \\
109,113,114, \\
117,119,121- \\
125\end{array}$ \\
\hline 218 & Bombus pomorum (Panzer, 1805) & $4 / 4$ & - & 25,78 & 102 \\
\hline 219 & Bombus pratorum (Linnaeus, 1761) & $50 / 23$ & 1,4 & $\begin{array}{l}25,30,47-49 \\
51,72,78\end{array}$ & $\begin{array}{l}101,103,106 \\
113,121-124\end{array}$ \\
\hline 220 & Bombus ruderarius (Müller, 1776) & $44 / 20$ & - & $\begin{array}{c}47-49,51,72 \\
74,76,77\end{array}$ & $\begin{array}{c}101,113,114, \\
117,121,123, \\
124\end{array}$ \\
\hline 221 & Bombus ruderatus (Fabricius, 1775) & $2 / 2$ & - & 47 & 121 \\
\hline 222 & Bombus subterraneus (Linnaeus, 1758) & $24 / 0$ & - & 72 & 114,123 \\
\hline 223 & Bombus sylvarum (Linnaeus, 1761) & $7 / 4$ & 8 & 49 & $101,113,122$ \\
\hline 224 & Bombus terrestris (Linnaeus, 1758) & $88^{*}$ & 8,12 & 49,77 & - \\
\hline 225 & Bombus veteranus (Fabricius, 1793) & $9 / 7$ & 4 & 49 & $\begin{array}{c}101,114,122 \\
124\end{array}$ \\
\hline 226 & Psithyrus barbutellus (Kirby, 1802) & $7 / 1$ & - & 78 & $\begin{array}{l}101,102,108 \\
122,123\end{array}$ \\
\hline 227 & Psithyrus bohemicus (Seidl, 1837) & $268 / 8$ & 4 & $\begin{array}{c}29,32,48,51 \\
64,72,74\end{array}$ & $\begin{array}{c}97,100,101, \\
104,106,109, \\
112,114,115, \\
119,121-123\end{array}$ \\
\hline 228 & Psithyrus campestris (Panzer, 1801) & $95 / 12$ & 4 & $\begin{array}{c}24,29,39,48 \\
49,51,72,78\end{array}$ & $\begin{array}{c}100-102,106, \\
108,114,121- \\
123\end{array}$ \\
\hline
\end{tabular}




\begin{tabular}{|c|c|c|c|c|c|}
\hline 1 & 2 & 3 & 4 & 5 & 6 \\
\hline 229 & $\begin{array}{l}\text { Psithyrus norvegicus Sparre-Schneider, } \\
1918\end{array}$ & $7 / 0$ & - & 72 & 98,123 \\
\hline 230 & Psithyrus rupestris (Fabricius, 1793) & $40 / 5$ & 4,8 & $32,51,78$ & $\begin{array}{c}100,114,121- \\
123\end{array}$ \\
\hline 231 & Psithyrus sylvestris Lepeletier, 1832 & $61 / 17$ & - & $32,48,72$ & $\begin{array}{l}100,106,109 \\
119,121-123\end{array}$ \\
\hline 232 & $\begin{array}{l}\text { Psithyrus vestalis (Geoffroy in Fourcroy, } \\
1785 \text { ) }\end{array}$ & $19 / 3$ & - & 49,78 & $\begin{array}{l}101,102,108 \\
114,121-123\end{array}$ \\
\hline 233 & Apis mellifera Linnaeus, 1758 & & & & \\
\hline
\end{tabular}

*see Banaszak \& Rasmont, 1994;

Date from other regions:

Kraków-Wieluń Upland: Andrena marginata Fabricius, 1776 (96)

Wielkopolska-Kujawy Lowland: Andrena chrysosceles (Kirby, 1802) (91), Andrena jakobi Perkins, 1931 (90), Dasypoda hirtipes (Harris, 1780) (89), Heriades truncorum (Linnaeus1758) (91), Chelostoma florisomne (Linnaeus, 1758) (90), Osmia brevicornis (Fabricius, 1798) (92), Osmia coerulescens (Linnaeus, 1758) (95), Megachile circumcincta (Kirby, 1802) (89), Coelioxys brevis Eversmann, 1852 (89), Nomada flavoguttata (Kirby, 1802) (93), Nomada moeschleri Alfken, 1913 (90), Nomada rufipes Fabricius, 1793 (89), Anthophora quadrimaculata (Panzer, 1806) (94), Anthophora retusa (Linnaeus, 1758) (91), Epeolus cruciger (Panzer, 1799) (89), Thyreus histrionicus (Illiger, 1806) (89), Bombus distinguendus (F. Morawitz, 1869) (95),

Two bumblebee species, Bombus distinguendus and B. ruderatus, were very rarely reported in the last 2-3 decades, so their localities recorded during this study are noteworthy. By contrast, $B$. humilis is quite common in some parts of Poland.

Thyreus histrionicus - recorded in 1944 (locality no. 40) and in the 1970s (no. 50). This seems interesting, because the species is a cleptoparasite of Anthophora quadrifasciata (Vil.), which has not been recorded in Poland for a long time. Its last record was $1 q$ caught on 3 Sep 1965 in Nowa Grobla near Mielec (Banaszak 1973).

It must be emphasized that some of the species infrequently caught near Łódź are only locally rare. In other parts of Poland some of them are quite common, e.g. Andrena fucata, A. helvola, A. humilis, A. ventralis, Evylaeus villosulus, and Andrena vaga. The last of the mentioned species is often abundant near Bydgoszcz and Poznań. In early spring it forms large local populations on sandy sites, e.g. on mineral hills near meadows, especially if bee forage plants, like willows (Salix sp.) and dandelions (Taraxacum sp.), are abundant there.

\section{List of species represented by 1-3 individuals}

Hylaeus gibbus: Niesułków Kolonia, 22 Jul 1993, ô.

H. gracilicornis: Cząstków-Zapusty, 6 Aug 1984, .

H. pictipes: Marianów, 4 Jul 1990, ㅇ; 'Wolbórka' reserve, 25 Jul 1980, ô; Łódź-Stoki, 14 Jul 1985, ㅇ.

H. punctatus: Łódź, ul. Banacha, 23 Jul 1986, ㅇ.

H. punctulatissimus: Rogów, 12 Jul 1977,, , on a wooden building.

H. rinki: Tomaszów Mazowiecki, Księża Góra, 22 Aug 1988, ․

Colletes fodiens: Łódź-Stoki, 26 Jun 1986, ô, sand pit.

C. succinctus: Bukowiec, 22 Aug 1986, ㅇ, moor (afforested in 1990s); Łódź-Stoki, 26 Aug 1984,,$; 25$ Aug 1985, , sand pit.

Andrena alfkenella: Łódź-Stoki, 22 Jul 1988, ô, sand pit.

A. apicata: 'Wolbórka' reserve, 9 May 1980, ; Pytowice, 1 May 1987, 9.

A. chrysosceles: Łódź - Botanical Garden, 28 Jul 1995, +, grassland; Daszyna, 26 May 1988, + , village park.

A. fucata: Łódź - Łagiewniki Forest, 25 Apr 1984, đ̊; 30 May 1988, q; 'Molenda' reserve, 12 Jul 1980, $q$. 
A. helvola: Wiączyń, 4 May 1984, †; Łódź - Łagiewniki Forest, 18 Apr 1986, @; Łódź-Stoki, 14 May 1986, ふ̊, sand pit.

A. humilis: Modlica, 14 Jun 1943, +; Kalonka, 16 May 1986, + ; Chełmowa Góra, 15 Jun 1984,, , meadow.

A. labialis: Uniejewo, 5 Jun 1988, Ô, slope of Vistula valley.

A. lapponica: 'Łaznów' reserve, 31 May 1991, ․ .

A. marginata: Działoszyn, 20 Aug 1976, o; Pińczów, 6 Aug 1976, o.

A. mitis: Łódź - Botanical Garden, 11 Jun 1980, ․

A. ovatula: Łódź - Łagiewniki Forest, 25 Aug 1987, ô; Łódź-Stoki, 4 Aug 1990, , , grassland; Nowa Słupia, 27 Aug 1980, ․

A. rosae: Cząstków-Zapusty, 14 Jul 1979, đ̂; 7 Aug 1979, ㅇ.

A. ruficrus: Modlica, 14 Jun 1943, 9 ; 9 Jun 1944, + .

A. schencki: Modlica, 6 Jun 1944, $\overbrace{}^{\lambda}$.

A. suerinensis: Łódź-Stoki, 5 Jun 1977, ô, sand pit.

A. synadelpha: Łódź-Stoki, 14 May 1989, , sand pit; Smardzew, 6 May 1990,.+

A. vaga: Bromierzyk, 1 May 1992, $\widehat{\jmath}$.

A. ventralis: Łódź - Łagiewniki Forest, 23 Apr 1984, ; Łódź-Radogoszcz, 23 Apr 1986, 2 우, near railway tracks.

A. wilkella: Marianów, 21 Jul 1988, + ; Dąbrowa, 8 Aug 1986, ․

Seladonia confusa: Barycz, 8 Jul 1943, ô; Dobryszyce, 14 Aug 1988, §̊, ㅇ. .

Lasioglossum lativentre: 'Babsk' reserve, 6 Sep 1988, ô; Biskupice, 13 Aug 1988, 우 Rawa Mazowiecka, 10 Sep 1988, ô, park.

L. prasinum: Osiny, 23 Aug 1990, .

L. subfasciatum: Łódź-Stoki, 22 Apr 1986, 2 q , sand pit; Święty Krzyż, 10 Aug 1983, ô.

L. xanthopus: Łódź-Stoki, 21 Jun 1977, 2 + ㅇ; Skowronno, 17 Jun 1972,.+

Evylaeus limbellus: Uniejewo, 9 Aug 1988,, .

E. lucidulus: Uniejewo, 9 Aug 1988, đ̊; Łódź - Botanical Garden, 17 Jul 1992, ㅇ.

E. minutissimus: Uroczysko Rawski Las, 25 Aug 1988, đ̊.

E. rufitarsis: Uniejewo, 16 Jun 1991, 9 , slope of Vistula valley.

E. semilucens: Łódź - Łagiewniki Forest, 9 Aug 1989, 2 ふึે.

E. tarsatus: 'Babsk' reserve, 12 Jul 1988, ㅇ; 15 Jul 1988, ㅇ․

E. villosulus: Tomaszów Mazowiecki - Księża Góra, 10 Jul 1989, +.

Rophites quinquespinosus: Łódź-Stoki, 28 Jul 1990, ㅇ, sand pit; Łódź-Stoki, 1 Aug 1990, 우 grassy slopes between fields; Skowronno, 7 Jun 1972, §.

Sphecodes ferruginatus: Łódź-Lublinek, 24 Aug 1976, †; Łódź-Stoki, 24 Aug 1976, O; 16 Aug 1985, ㅇ, sand pit.

S. marginatus: Tomaszów Mazowiecki - Księża Góra, 12 Aug 1989, ㅇ.

S. puncticeps: Łódź-Stoki, 16 Aug 1985, ô, sand pit.

Melitta haemorrhoidalis: Grzegorzowice - 'Wąwóz w Skałach' reserve, 9 Aug 1984, .

Stelis minuta: Dobieszków, 2 Jul 1991, ㅇ.

S. ornatula: Łódź - Park 3-go Maja, 13 Jun 1987, ठ̂.

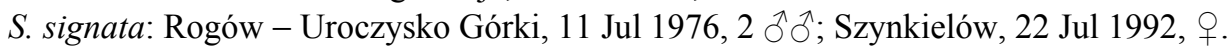

Chelostoma distinctum: Marianów, 25 Aug 1990, ㅇ.

Hoplitis anthocopoides: Szynkielów, 14 Jul 1992, ㅇ.

H. leucomelana: Dobryszyce, 14 Aug 1988, , ; Łódź-Stoki, 31 Aug 1988, q, sand pit.

Osmia leaiana: Tuszyn-Poddębina, 27 Jul 1980, ô, wooden building; Gałków, 20 Jun 1990, + , near railway tracks; Grzegorzowice, 9 Aug 1984,, , meadow. 
O. mustelina: Łódź-Stoki, 22 Jun 1985, , sand pit; Łódź - Park Źródliska, 17 Jun 1986, , didactic garden; Łódź - ul. Giewont, 8 Jun 1986, §̋, wooden building.

O. uncinata: Smardzew, 6 May 1990, q, flowering hawthorn.

Megachile pilidens: Bromierzyk, 5 Aug 1992, ô; Łódź - Botanical Garden, 9 Jul 1992, O+; Szynkielów, 14 Jul 1992, ત̃.

Coelioxys aurolimbata: Łódź - Park Źródliska, 17 Jun 1986, đ̊, didactic garden.

C. elongata: Bromierzyk, 7 Aug 1991, , , road among fields; Łódź-Stoki, 28 Jul 1991, ô, sand pit.

C. inermis: Łódź-Stoki, 8 Sep 1984, + , sand pit.

C. rufocaudata: 'Popień' reserve, 26 Jul 1976, + ; Tomaszów Mazowiecki - Księża Góra, 25 Jun 1989, ô.

Nomada femoralis: Łódź-Stoki, 29 May 1985, 3 우, afforested former farmland.

N. fuscicornis: Łódź - Łagiewniki Forest, 8 Aug 1987, đ゙̄, side of forest road; Łódź-Stoki, 31 Jul 1988, + , sand pit; Łódź-Stoki, 1 Aug 1990, , grassy slopes between fields.

N. panzeri: Łódź-Stoki, 26 Apr 1981, ô, sand pit; Łódź-Stoki, 14 May 1992, + , green areas between apartment blocks.

N. sexfasciata: Rogów, 28 Jun 1975, q; Michałów, 5 Jul 1976, q; Glinnik, 3 Jun 1984, q, cmentarz. Anthophora plagiata: Łódź - Park Źródliska, 17 Jun 1986, §̃, didactic garden.

A. retusa: Łódź-Stoki, 18 May 1976, + (melanotic individual), grassy slopes between fields; Łódź-Stoki, 11 May 1985, ô, afforested former farmland; Daszyna, 26 May 1988, ふૈ, gravel mine.

Biastes emarginatus: Łódź-Stoki, 8 Jul 1990,, , sand pit.

Epeoloides coecutiens: Szynkielów, 22 Jul 1992, đ̂, mire; Góra Radostowa, 9 Jul 1981, đ̊, meadow.

Thyreus histrionicus: Justynów, 27 Jul 1944, + ; Michałów, 14 Aug 1974, đ̊; Burzenin, 10 Aug 1973, ㅇ․

T. orbatus: Bromierzyk, 5 Aug 1992, Ô, meadow; Ludwików, 10 Jul 1989, ๆ; Łódź-Stoki, 28 Jul 1991, ڤ̂, sand pit.

Bombus distinguendus: Gałków, 3 May 1987, +, near railway tracks; 'Winnica' reserve, 14 Jul 1992, ô.

B humilis: Szewna, 6 Sep 1979, , loess ravines; Cząstków-Zapusty, 30 Jun 1979, +, worker, on flowers of Astragalus sp.

B. ruderatus: Łódź - Botanical Garden, 24 Apr 1986, ; Szklana Huta, 16 Aug 1979, o.

\section{SUMMARY AND CONCLUSIONS}

This study fills an important gap in the current knowledge of the bee fauna (Apiformes) in Poland, as it provides data on the distribution of bees in central Poland. A total of 233 bee species were recorded in the study area, including 218 in the northwestern part of the Małopolska Upland (mainly on the Łódź Hills), 89 in the Świętokrzyskie Mts., and 49 in the Vistula valley near Płock. In the area of Łódź Hills, the most common were Heriades truncorum (4.3\% of individuals caught there) and Hylaeus communis (2.7\%), while in the Świętokrzyskie Mts., bumblebees and cuckoo bees were the most abundant: Psithyrus bohemicus (6.8\%), Bombus lucorum (4.2\%), Bombus pascuorum (3.7\%), B. lapidarius (2.4\%) and Psithyrus campestris $(2.4 \%)$.

The collected material includes 71 rare species (over $30 \%$ of all species recorded in this study). Particularly noteworthy are: Hylaeus gredleri, Andrena suerinensis, Lasioglossum prasinum, Evylaeus limbellus, E. semilucens, E. tarsatus, Osmia mustelina, O. uncinata, Anthophora plagiata and Thyreus histrionicus. Some of the recorded taxa have become locally rare recently: Andrena vaga, A. fucata, A. helvola, A. humilis, A. ventralis and Evylaeus villosulus. 


\section{ACKNOWLEDGMENTS}

We are grateful to E. Ambrozik, B. Gałczyńska, M. Kobalczyk, J. Kurzawa, E. Kwiatkowska, A. Liana, J. Mańkowska, M. Mastalerz, Z. Myślicka, J. Nadolski, J. Sobczak, B. Soszyński, E. Szczepański, J. Szczypciak, Z. Śliwiński, M. Wanat, C. Watała and A. Zborowska, who kindly provided some bee specimens from the study area. We also thank Mr. T. Kurzac, who greatly helped us in the preparation of this paper for publication.

\section{REFERENCES}

Abraszewska-KowalczyK A., KowalczyK J. K., Hejduk J., PrZybylski M. \& Tuszewicki W. 2002. Świat zwierzęcy Brudzeńskiego Parku Krajobrazowego. Mantis, Olsztyn, 101 pp.

BANASZAK J. 1973. Materiały do znajomości fauny pszczołowatych (Hymenoptera, Apoidea) Rzeszowszczyzny. Polskie Pismo Entomol. 43: 55-60.

BANASZAK J. 1982. Pszczoły (Apoidea, Hymenoptera) Niziny Wielkopolsko-Kujawskiej. Fragmenta Faunistica 27: 75-92.

Banaszak J. 2004. Apidae. In: Bogdanowicz W., Chudzicka E., Pilipiuk I. \& Skibińska E. (eds), Fauna of Poland. I., pp. 358-362. Museum and Institute of Zoology PAS, Warszawa.

BANASZAK J. \& RASMONT P. 1994. Occurrence and distribution of the subgenus Bombus Latreille sensu stricto in Poland (Hymenoptera, Apoidea). Polskie Pismo Entomologiczne 63: 337-356.

BanaszaK J. \& PlewKA T. 1981. Apoidea (Hymenoptera) Kampinoskiego Parku Narodowego. Fragmenta Faunistica 25: 435-452.

BANASZAK W. A. 2003. Rozmieszczenie i ocena zagrożeń występowania w Polsce Anthophora plagiata (Illiger 1806) (Hymenoptera: Apoidea). Nowy Pamiętnik Fizjograficzny 2: 53-68.

BANASZAK W. A. 2005. What has caused the decline of the solitary bee Anthophora plagiata (Illiger, 1806) (Hymenoptera: Apoidea) in the Wielkopolska-Kujawy Lowland in west Poland? Polskie Pismo Entomologiczne 74: $157-185$.

BĄK J. 1998. Trzmiele (Bombus Latr.) (Hymenoptera, Apidae) na terenie rezerwatu „Cisów” w Górach Świętokrzyskich. In the conference material: Przemiany środowiska geograficznego obszarów górskich w Polsce i jego stan współczesny, Wólka Milanowska, 26-27. 10. 1998, Instytut Geografii Wyższej Szkoły Pedagogicznej, Komitet Zagospodarowania Ziem Górskich, pp. 16-19, Kielce.

BĄK J. 1999a. Skład gatunkowy i struktura dominacji trzmieli (Bombus spp.) (Hymenoptera: Apoidea) rezerwatu „Białe Lugi” w Górach Świętokrzyskich. In the conference material: Ochrona owadów w Polsce u progu integracji z Unią Europejską. Konferencja Naukowa, Kraków 23-24. 09. 1999, p. 1. Poznań-Kraków.

BĄK J. 1999b. Nowe stanowisko Bombus magnus Vogt, 1911 (Hymenoptera, Apidae) w Polsce. Rocznik Świętokrzyski, ser. B, 26: 71-72.

BĄK J. 2003. Trzmiele (Bombus Latr.) i trzmielce (Psithyrus Lep.) (Hymenoptera, Apidae) w wybranych fitocenozach Suchedniowsko-Oblęgorskiego Parku Krajobrazowego w Górach Świętokrzyskich. Prądnik. Prace Muzeum Szafera 13: 235-244.

ĆMAK J. \& SzCZYPCIAK-BĄK J. 1987. Trzmiele (Bomus Latr.) w biotopach Świętokrzyskiego Parku Narodowego. Studia Kieleckie 4: 101-112.

DrogoszewsKi K. 1932. Wykaz żądłówek zebranych w Polsce Środkowej. Pol. Pismo Entomol., Lwów, 11: $113-118$.

Drogoszewski K. 1934. Nowe dla Polski Środkowej żądłówki. Polskie Pismo Entomologiczne, Lwów, 13: 125-130.

DylewsKa M. 2000. Pszczołowate - Apidae, Podrodzina Andreninae. Klucze do oznaczania owadów Polski. Część XXIV. Błonkówki - Hymenoptera. Polskie Towarzystwo Entomologiczne. Toruń, 152 pp.

DYlewSKA M. \& BĄK J. 2005. Apiformes (Hymenoptera, Apoidea) of the Lysogóry Mountains and adjacent area. Acta Zoologica Cracoviensia 48 B: 145-179.

Dylewska M., Gąsienica-Chmiel M., Kosior A., Sumera B., Szafraniec S., Werstak K. \& WiŚniowski B. 1998. Skład gatunkowy i liczebność trzmieli i trzmielców (Bombinae, Apoidae, Hymenoptera) na łąkach w wybranych parkach narodowych oraz kwiecistość łąk w tych parkach w 1998 roku. Prądnik. Prace Muzeum Szafera 11-12: 279-292.

KOWALCZYK J. K. 1996a. Bezkręgowce lądowe Ogrodu Botanicznego w Łodzi. In: KuRZAC T. (ed.), Przyroda Ogrodu Botanicznego w Łodzi, pp. 141-157. Sagalara, Łódź.

KOWALCZYK J. K. 1996b. O ochronę siedlisk owadów leśnych w zieleni miejskiej Łodzi. Chrońmy Przyrodę Ojczystą 52: 106-107.

KowALCZYK J. K. 1997. Nowe stanowiska żądłówek (Hymenoptera: Aculeata) rzadko spotykane w Polsce. Wiadomości entomologiczne 16: 53-54.

KOWALCZYK J. K. \& KURZAC T. 2002. Wstępna lista wybranych rodzin błonkówek (Hymenoptera) i muchówek (Diptera) Załęczańskiego Parku Krajobrazowego. In: KUROWSKI J. K. \& WiTOSŁAWSKI P. (eds), Funkcjonowanie 
Parków Krajobrazowych w Polsce, pp. 147-151. Katedra Geobotaniki i Ekologii Roślin, Uniwersytet Łódzki, Łódź.

KowalczyK J. K. \& KurZaC T. 2005. Żądłówki (Hymenoptera, Aculeata) Ogrodu Botanicznego w Łodzi. Biuletyn Ogrodów Botanicznych 14: 101-113.

KowAlCZYK J. K., MAJECKi J., SOSZYŃSKi B. \& ŚLIWIŃSKi Z. 1998. Świat zwierzęcy. In: KuROwSKi J. K. (ed.), Park Krajobrazowy Wzniesień Łódzkich, pp. 81-96. Wojewódzki Fundusz Ochrony Środowiska i Gospodarki Wodnej w Łodzi. Eko-Wynik, Łódź.

KowalczyK J. K., Szczepko K., KurZaC T. \& PAwlikowski T. 2004. Pszczoły (Hymenoptera, Apoidea) Ogrodu Botanicznego w Łodzi. In: Indykiewicz P. \& Barczak T. (eds), Fauna miast Europy Środkowej 21. wieku, pp. 6782. Wyd. LOGO, Bydgoszcz.

KowALCZYK J. K. \& ŚLIWIŃSKi Z. 1988. Uwagi o entomofaunie lądowej Świętokrzyskiego Parku Narodowego. Parki Narodowe i Rezerwaty Przyrody 8: 33-39.

KowAlCZYK J. K. \& WATAŁA C. 1987. Interesujące gatunki żądłówek (Hymenoptera, Aculeata) na Wyżynie Łódzkiej. Przegląd Zoologiczny 31: 59-62.

KowalczyK J. K. \& Watała C. 1990. Przyczynek do poznania entomofauny projektowanego SuchedniowskoOblęgorskiego Parku Krajobrazowego. Rocznik Świętokrzyski, Kielce, 17: 173-180.

ŁOZIŃSKI J. 1920. Błonkówki pszczołowate okolic Krakowa. Sprawozdania Komisji Fizjograficznej 53/54: $125-135$.

OsytshnjuK A. Z., Romasenko L., BANASZAK J. \& CierzNiaK T. 2005. Andreninae of the Central and Eastern Palaearctic. Part 1. Polish Entomological Monographs, 2. Polish Entomological Society, Poznań-Bydgoszcz, 235 pp.

Pesenko Yu., Banaszak J., Radchenko V.G. \& CierzniaK T. 2000. Bees of the family Halictidae (excluding Sphecodes) of Poland: taxonomy, ecology, bionomics. Wydawnictwa Uczelniane Wyższej Szkoły Pedagogicznej w Bydgoszczy, Bydgoszcz, 348 pp.

Ruszkowski A., BilińSKi M., Kosior A., BĄK J. \& KACZMARsKa K. 1989. Trzmiele Wyżyny Małopolskiej. Pszczelnicze Zeszyty Naukowe, Puławy, 33: 55-76.

SZCZEPKO K., KOWALCZYK J. K. \& NADOLSKI J. 2004. Stan poznania oraz próba wstępnej waloryzacji żądłówek (Hymenoptera, Aculeata) Łodzi. In: INDYKIEWICZ P. \& BARCZAK T. (eds), Fauna miast Europy Środkowej 21. wieku, pp. 49-65. Logo, Bydgoszcz.

SZCZEPKO K., PAWLIKOWSKi T. \& KOWALCZYK J. K. 2002. Apoidea (Hymenoptera) in habitats of former agriculture area in renaturalization stage of Kampinos National Park (Poland). Fragmenta Faunistica 45: 115-122.

ZARĘBA R. 1981. Puszcze, bory i lasy Polski. PWRiL, Warszawa, 187 pp.

\section{STRESZCZENIE}

\section{[Materiały do znajomości pszczół środkowej Polski (Hymenoptera: Apoidea: Apiformes)]}

Praca wypełnia poważną lukę w poznaniu fauny pszczół Apiformes Polski, jaką stanowiła centralna część kraju. Ogółem wykazano 233 gatunki Apiformes, z tej liczby z Wyżyny Małopolskiej (głównie Wzniesienia Łódzkie) - 218 gatunków, z Gór Świętokrzyskich - 89 i z Doliny Wisły pod Płockiem - 49 gatunków.

Do najliczniejszych gatunków Wzniesień Łódzkich należały Heriades truncorum $(4,3 \%)$ i Hylaeus communis (2,7\%), natomiast w Górach Świętokrzyskich dominują ilościowo trzmiele i ich pasożyty: Bombus lucorum (4,2\%) i Psithyrus bohemicus (6,8\%). Tam też liczne były: Bombus pascuorum (3,7\%), B. lapidarius (4\%) i Psithyrus campestris $(2,4 \%)$.

W omawianym materiale 71 gatunków (ponad 30\%) to taksony rzadkie. Spośród nich na szczególną uwagę zasługują: Hyaleus gredleri, Andrena suerinensis, Lasioglossum prasinum, Evylaeus limbellus, E. semilucens, E. tarsatus, Osmia mustelina, O. uncinata, Anthophora plagiata i Thyreus histrionicus. Do gatunków lokalnie rzadkich w ostatnich latach należy Andrena vaga, A. fucata, A. helvola, A. humilis, A. ventralis i Evylaeus villosulus. 